\title{
On the Critical Points of the Structure Amplitude for the Case of One-Dimensional Displacive Modulation
}

\author{
T. Śliwińska, P. Gusin And J. Warczewski \\ Institute of Physics, University of Silesia \\ Uniwersytecka 4, 40-007 Katowice, Poland
}

(Received November 12, 2004; revised version January 31, 2005)

\begin{abstract}
The behavior of the structure amplitude is analyzed in terms of the harmonics of a modulation function for the case of one-dimensional longitudinal displacive modulation. This modulation function is given by a finite series of $N$ sine functions. It turns out that: (1) for the large values of the amplitudes of the modulation function the structure amplitude tends to zero for the arbitrary values of the order $m$ of the satellite reflections; (2) for certain values of the amplitudes of the modulation function the modulus of the structure amplitude assumes its maximum value and (3) for the latter case the intensity of an arbitrary satellite reflection is a function of the intensities of both the closest main reflection and the intensities of the finite set of the neighbor satellite reflections.
\end{abstract}

PACS numbers: 61.44.Fw, 61.10.Dp

\section{Introduction}

Lattice periodicity (translational symmetry) is a property that is considered as an essential concept in crystallography. For the aperiodic structures an embedding procedure into the higher-dimensional space is applied in order to recover the periodicity of these structures, the latter property makes it possible to construct the corresponding (super)space symmetry groups. In recent decades more and more modulated crystal phases have been observed in nature. Progress in the symmetry description of modulated structures with the aid of higher-dimensional crystallographic symmetry groups has been obtained as a result of many important papers (e.g. [1]). In the case of the structure with the displacive modulation 
the positional vector of the $\mu$-th atom $\boldsymbol{R}_{\mu}$ can be expressed as periodic functions of the components $\boldsymbol{q}^{a} \cdot \widehat{\boldsymbol{r}}_{\mu}$ of the modulation vector, where $a=1, \ldots, d$ ( $d$ being the modulation dimension) as follows:

$$
\boldsymbol{R}_{\mu}=\widehat{\boldsymbol{r}}_{\mu}+\boldsymbol{u}_{\mu}\left(\boldsymbol{q}^{1} \cdot \widehat{\boldsymbol{r}}_{\mu}, \ldots, \boldsymbol{q}^{d} \cdot \widehat{\boldsymbol{r}}_{\mu}\right),
$$

where $\widehat{\boldsymbol{r}}_{\mu}=\boldsymbol{p}+\boldsymbol{r}_{\mu}$ and $\boldsymbol{p}$ is a lattice vector and $\boldsymbol{r}_{\mu}$ is a position vector of $\mu$-th atom in an elementary cell, $\boldsymbol{u}_{\mu}$ is the modulation function of the $\mu$-th atom with the components

$$
u_{\mu \alpha}\left(t+\boldsymbol{q} \cdot \boldsymbol{r}_{\mu}\right)=\sum_{n=1}^{N} u_{\alpha n}^{(\mu)} \sin \left[n\left(t+\boldsymbol{q} \cdot \boldsymbol{r}_{\mu}\right)+\varphi_{\mu \alpha}(n)\right],
$$

$t=\boldsymbol{q} \cdot \boldsymbol{p}$ and $\mu$ enumerates the independent atoms, $N$ is the number of harmonics, $\alpha=1,2,3$, whereas $\varphi_{\mu \alpha}(n)$ is a phase parameter, which depends on $\mu$ and $n$.

In the case of one-dimensional modulation the modulation vector $\boldsymbol{q}$ is related to the diffraction pattern in the following way:

$$
\boldsymbol{H}=2 \pi h \boldsymbol{a}^{*}+2 \pi k \boldsymbol{b}^{*}+2 \pi l \boldsymbol{c}^{*}+m \boldsymbol{q},
$$

where $\boldsymbol{a}^{*}, \boldsymbol{b}^{*}, \boldsymbol{c}^{*}$ are the reciprocal-lattice vectors of the basic structure. (We use the following scalar product between basic vectors: $\boldsymbol{a} \cdot \boldsymbol{a}^{*}=\boldsymbol{b} \cdot \boldsymbol{b}^{*}=\boldsymbol{c} \cdot \boldsymbol{c}^{*}=1$.) In the diffraction pattern of such a modulated phase, one can distinguish the main reflections (for which $m=0$ ) and satellites (for which $m \neq 0$ ). The satellites appear in the points of the reciprocal lattice described by Eq. (3). In the model under consideration the modulation function is a periodic function for which a harmonic approximation is used (Eq. (2)). The crystals with the diffraction vector defined in Eq. (3) need for their description $3+1$ basic vectors in the reciprocal lattice. De Wolff, Janssen, and Janner (1981) present the list of (3+1)-dimensional superspace groups describing the incommensurate crystal structures with one-dimensional modulation.

In Ref. [2] there is studied the sensitivity of X-ray diffraction to the structural differences in an incommensurate structure between the sinusoidal and soliton regime. The case of the small amplitudes of the expansion of the displacive modulation function as well as the one-harmonic approximation of the modulation function has been studied in [3]. In Refs. [4, 5] Aramburu et al. have studied for $\mathrm{Rb}_{2} \mathrm{ZnCl}_{4}$ the temperature dependence of intensities of both the main reflections and the satellite reflections. Böhm [6] has studied the behavior of the rectangular and triangular modulations to compare their effect with the sinusoidal modulation.

The aim of the present paper is to study the behavior of the modulated structure amplitude from the point of view of the monotonicity of its modulus for the case of one-dimensional longitudinal displacive modulation. The harmonic expansion of a modulation function is here assumed with the following conditions concerning the phases as well as the amplitudes of the modulation function: 


$$
\begin{aligned}
& \forall n, \mu \varphi_{\mu \alpha}(n)=\varphi_{\alpha}, \\
& \forall \mu u_{\alpha n}^{(\mu)}=u_{\alpha n} .
\end{aligned}
$$

Under the above conditions the modulation function has the form

$$
u_{\alpha}\left(t+\boldsymbol{q} \cdot \boldsymbol{r}_{\mu}\right)=\sum_{n=1}^{N} u_{\alpha n} \sin \left[n\left(t+\boldsymbol{q} \cdot \boldsymbol{r}_{\mu}\right)+\varphi_{\alpha}\right] .
$$

Under the assumption of a displacive modulation the function describing the probability that an atom is in a modulated position, can be equal to either 0 or 1 . With such a homogeneous probability distribution the formula of the modulated structure amplitude $F(\boldsymbol{H}, m)$ for the modulation function given by Eq. (6) has the form $[7-10]$ :

$$
\begin{gathered}
F(\boldsymbol{H}, m)=\sum_{\mu} f_{\mu}(\boldsymbol{H}) \exp \left[\mathrm{i}(\boldsymbol{H}-m \boldsymbol{q}) \cdot \boldsymbol{r}_{\mu}\right] \\
\times \int_{0}^{2 \pi} \exp (\mathrm{i} m x) \exp [\mathrm{i} \boldsymbol{H} \cdot \boldsymbol{u}(x)] \mathrm{d} x
\end{gathered}
$$

where $x=t+\boldsymbol{q} \cdot \boldsymbol{r}_{\mu}$. The above equation can be rewritten in the following form:

$$
\begin{aligned}
& F(\boldsymbol{H}, m)=\frac{1}{2 \pi} \sum_{\mu} f_{\mu}(\boldsymbol{H}) \exp \left[\mathrm{i}(\boldsymbol{H}-m \boldsymbol{q}) \cdot \boldsymbol{r}_{\mu}\right] \\
& \times \int_{0}^{2 \pi} \exp (\mathrm{i} m x) \exp \left[\mathrm{i} \sum_{n=1}^{N} z_{n} \sin \left(n x+\xi_{n}\right)\right] \mathrm{d} x
\end{aligned}
$$

where

$$
\begin{aligned}
& z_{n}=\left[\left(H_{1} u_{1 n}\right)^{2}+\left(H_{2} u_{2 n}\right)^{2}+\left(H_{3} u_{3 n}\right)^{2}+2 H_{1} u_{1 n} H_{2} u_{2 n} \cos \left(\varphi_{1}-\varphi_{2}\right)\right. \\
& \left.\quad+2 H_{1} u_{1 n} H_{3} u_{3 n} \cos \left(\varphi_{1}-\varphi_{3}\right)+2 H_{2} u_{2 n} H_{3} u_{3 n} \cos \left(\varphi_{2}-\varphi_{3}\right)\right]^{\frac{1}{2}}, \\
& \sin \xi_{n}=\frac{1}{z_{n}} \sum_{\alpha=1}^{3} H_{\alpha} u_{\alpha n} \cos \varphi_{\alpha}, \\
& \cos \xi_{n}=\frac{1}{z_{n}} \sum_{\alpha=1}^{3} H_{\alpha} u_{\alpha n} \sin \varphi_{\alpha}, \\
& H_{1}=2 \pi h+m q_{1}, \quad H_{2}=2 \pi k+m q_{2}, \quad H_{3}=2 \pi l+m q_{3},
\end{aligned}
$$
$n=1, \ldots, N, d=\left(d_{1}, \ldots, d_{N}\right), \xi=\left(\xi_{1}, \ldots, \xi_{N}\right)$ and $u_{\alpha n}$ are defined by Eq. (6). 
Another form of the structure amplitude of the modulated structure can be obtained by integrating Eq. (8) over $x$. This procedure leads among others to the summation of products of the Bessel functions

$$
\begin{aligned}
& F(\boldsymbol{H}, m)=\sum_{\mu} f_{\mu}(\boldsymbol{H}) \exp \left[\mathrm{i}(\boldsymbol{H}-m \boldsymbol{q}) \cdot \boldsymbol{r}_{\mu}\right] \sum_{\boldsymbol{d} \in \boldsymbol{Z}^{N}} J_{d_{1}}\left(z_{1}\right) \ldots J_{d_{N}}\left(z_{N}\right) \\
& \quad \times \exp (\mathrm{i} \boldsymbol{d} \cdot \boldsymbol{\xi}) \delta\left(m+\sum_{l=1}^{N} l d_{l}, 0\right) .
\end{aligned}
$$

It is easy to see that the structure amplitude given by Eq. (13) does not vanish under the following condition:

$$
m+\sum_{l=1}^{N} l d_{l}=0 .
$$

This paper is organized as follows: in Sect. 2 the analysis of Eqs. (8) and (13) describing the displacive modulated structure amplitude expressed by the Bessel functions is carried out. Two problems among others are discussed: (1) an asymptotic expansion of the modulated structure amplitude for high values of $z_{n}$, (2) a relationship between the maximum value of the module of the structure amplitude and the values of the amplitudes of the modulation function. In Sect. 3 one-harmonic modulation is considered. Some computations as well as the method of a stationary phase are presented in Appendices.

\section{Analysis of the formula for the modulated structure amplitude}

Let us consider an asymptotic expansion of the modulated structure amplitude (Eq. (8)) for high values of $z_{n}$. Because $z_{n}$ are related to $u_{\alpha n}$ by Eq. (9), one can say that this case concerns the behavior of the structure amplitude at large values of the amplitudes of the expansion of the modulation function. It is assumed in this model that these amplitudes should not be too high because the crystal structure itself has to be maintained [6]. However, it remains still interesting whether the modulus of the structure amplitude assumes a maximum value in the physical interval of values of the amplitudes of the modulation function. At this moment we know only that the modulus under consideration increases from zero with $z_{n}$ at the very low values of $z_{n}$. Therefore the next step in our considerations is to study the behavior of this modulus as a function of $z_{n}$ for higher $z_{n}$ (asymptotic expansion).

\subsection{Asymptotic expansion of the modulated structure amplitude for large values of $z_{n}$}

As according to Eq. (9) $z_{n}$ is a function of $\boldsymbol{H}$, the structure amplitude (see Eq. (8)) can be rewritten for a given satellite order $m$ as follows: 


$$
F\left(z_{1}, \ldots, z_{N} ; m\right)=S(\boldsymbol{H}, m) g\left(z_{1}, \ldots, z_{N} ; m\right)
$$

where

$$
\begin{aligned}
& S(\boldsymbol{H}, m)=\sum_{\mu} f_{\mu}(\boldsymbol{H}) \exp \left[\mathrm{i}(\boldsymbol{H}-m \boldsymbol{q}) \cdot \boldsymbol{r}_{\mu}\right] \\
& g\left(z_{1}, \ldots, z_{N} ; m\right)=\int_{0}^{2 \pi} \exp (\mathrm{i} m x) \exp \left[\mathrm{i} \sum_{n=1}^{N} z_{n} \sin \left(n x+\xi_{n}\right)\right] \mathrm{d} x
\end{aligned}
$$

and $z_{n}$ is defined by Eq. (9). From the last two formulas it is easy to see that only the factor $g$ undergoes the procedure of the asymptotic expansion.

Let us assume that $z_{a} \rightarrow \infty(a=1, \ldots, N)$. The method of a stationary phase (see Appendix A.1) brings us to the following formula:

$$
\begin{aligned}
& g\left(z_{1}, \ldots, z_{a}, \ldots, z_{N} ; m\right) \underset{z_{a}}{\simeq} \rightarrow \infty \frac{1}{a} \sqrt{\frac{2 \pi}{z_{a}}} \\
& \quad \times \exp \left[\mathrm{i}\left(z_{a}+(2 m-a) \pi / 4-m \xi_{a} / 2 a+\sum_{n \neq a}^{N} z_{n} \Phi_{n}\left(x_{a}\right)\right)\right],
\end{aligned}
$$

where

$$
\begin{aligned}
& x_{a}=\left(\pi-\xi_{a}\right) / 2 a, \\
& \Phi_{n}(x)=\sin \left(n x-\xi_{n}\right) .
\end{aligned}
$$

Parameter $z_{a}$ is a function of $a$-th amplitude $u_{a}$, of wave vector $\boldsymbol{H}$ and of angles $\varphi_{1}, \varphi_{2}, \varphi_{3}$. For fixed $\boldsymbol{H}$ parameter $z_{a}$ goes to infinity with the $a$-th amplitude of the modulation function. Thus the asymptotic form of the structure amplitude is

$$
\begin{aligned}
& F\left(z_{1}, \ldots, z_{a}, \ldots, z_{N} ; m\right) \underset{u_{\alpha a} \rightarrow \infty}{\simeq} \sum_{\mu} f_{\mu}(\boldsymbol{H}) \exp \left[\mathrm{i}(\boldsymbol{H}-m \boldsymbol{q}) \cdot \boldsymbol{r}_{\mu}\right] \\
& \times \frac{1}{a} \sqrt{\frac{2 \pi}{z_{a}}} \exp \left\{\mathrm{i}\left[z_{a}+(2 m-a) \pi / 4-m \xi_{a} / 2 a\right]+\sum_{n \neq a}^{N} z_{n} \Phi_{n}\left(x_{a}\right)\right\} .
\end{aligned}
$$

As it follows from the above formula, one can say that if the amplitudes of the modulation function go to infinity, then the modulus of the structure amplitude goes to zero. Thus

$$
\left|F\left(z_{1}, \ldots, z_{a}, \ldots, z_{N} ; m\right)\right|_{u_{\alpha a} \rightarrow \infty}^{\rightarrow} 0 .
$$

On the other hand, if all the amplitudes of the modulation function go to zero, then the module of the structure amplitude assumes the following form (see Appendix A.3): 


$$
\left|F\left(z_{1}, \ldots, z_{a}, \ldots, z_{N} ; m\right)\right|_{u_{\alpha 1}, \ldots, u_{\alpha N} \rightarrow 0}|S(\boldsymbol{k}, m)| \delta_{m, 0} .
$$

Let us note that for $m=0$ we deal with the nonmodulated structure, whereas for $m \neq 0$ the modulus of the structure amplitude is equal to zero $\left(\delta_{m, 0}\right.$ is the Kronecker delta).

\subsection{The maximum value of the modulus of the modulated structure amplitude}

As the structure amplitude goes to zero both for low and large values of the amplitudes $u_{\alpha n}$ of the modulation function, one can expect that for certain $u_{\alpha n}$ values the modulus of the structure amplitude assumes its maximum value. To find this maximum one has to differentiate $F$ (see Eq. (15)) with respect to $z_{n}$ and solve the following equations with respect to $z_{n}$ :

$$
\frac{\partial F}{\partial z_{n}}=0
$$

where $n=1, \ldots, N$. As a result of this differentiation and taking into account the following identity:

$$
\sin v=\frac{1}{2 \mathrm{i}}\left(\mathrm{e}^{\mathrm{i} v}-\mathrm{e}^{-\mathrm{i} v}\right),
$$

one obtains the $N$ equations (on $z_{n}$ ) which correspond to this maximum

$$
\begin{gathered}
\mathrm{e}^{2 \mathrm{i} \xi_{1}} g(\boldsymbol{z}, m+1)=g(\boldsymbol{z}, m-1), \\
\vdots \\
\mathrm{e}^{2 \mathrm{i} \xi_{N}} g(\boldsymbol{z}, m+N)=g(\boldsymbol{z}, m-N),
\end{gathered}
$$

where $\boldsymbol{z}=\left(z_{1}, \ldots, z_{N}\right)$. Let us denote by $\boldsymbol{z}_{0}$ one of the solutions of the above system of equations. Let us note that the number of such solutions is infinite. Let us try to find in this set of solutions a physical solution for the one-harmonic case.

The structure amplitude for $\boldsymbol{z}=\boldsymbol{z}_{0}$ obeys the relation

$$
F\left(\boldsymbol{z}_{0}, m+a\right)=\mathrm{e}^{-2 \mathrm{i} \xi_{a}} S(\boldsymbol{H}, m+a) g\left(\boldsymbol{z}_{0}, m-a\right),
$$

where $F(\boldsymbol{z}, m)=S(m) g(\boldsymbol{z}, m)$. Thus from the above relation one obtains the following recurrence formula:

$$
\frac{F\left(\boldsymbol{z}_{0}, m+a\right)}{F\left(\boldsymbol{z}_{0}, m-a\right)}=\frac{S(\boldsymbol{H}, m+a)}{S(\boldsymbol{H}, m-a)} \mathrm{e}^{-2 \mathrm{i} \xi_{a}}
$$

for $a=1, \ldots, N$. The general solution of this recurrence equation assumes the following form (see Appendix A.2):

$$
F\left(\boldsymbol{z}_{0}, 2 j m+i\right)=\prod_{l=1}^{m} \frac{S(\boldsymbol{H}, 2 j l+i)}{S(\boldsymbol{H}, 2 j l-2 j+i)} \exp \left(-2 \mathrm{i} \xi_{j}\right) F\left(\boldsymbol{z}_{0}, i\right),
$$

where $m>0, i=0, \ldots, 2 H-1 ; H=1, \ldots, N ; j=1, \ldots, N ; m=H, H+1, \ldots$ Thus the structure amplitude $F\left(\boldsymbol{z}_{0}, 2 j m+i\right)$ is expressed here by the finite series 
of the structure amplitude $F\left(\boldsymbol{z}_{0}, 0\right), \ldots, F\left(\boldsymbol{z}_{0}, i\right)$. In order to illustrate the above results let us consider in the next section an example.

\section{Structure amplitude in the case of one-dimensional modulation with one harmonic}

In the one-harmonic case of the modulation function (the vector $\boldsymbol{z}$ has only one component equal to $z$ ) the recurrence Eq. (27) assumes the following form:

$$
F\left(z_{0}, m+1\right)=\frac{S(\boldsymbol{H}, m+1)}{S(\boldsymbol{H}, m-1)} \mathrm{e}^{-2 \mathrm{i} \xi} F\left(z_{0}, m-1\right)
$$

where $z_{0}$ is a solution of the equation

$$
\mathrm{e}^{2 \mathrm{i} \xi} g(z, m+1)=g(z, m-1) .
$$

The solution of this recurrence equation is given by Eq. (28) and in this case has the form (for $m>0$ ):

$$
\begin{aligned}
& F\left(z_{0}, 2 m\right)=\frac{S(\boldsymbol{H}, 2 m)}{S(\boldsymbol{H}, 0)} \mathrm{e}^{-2 \mathrm{i} \xi} F\left(z_{0}, 0\right), \\
& F\left(z_{0}, 2 m+1\right)=\frac{S(\boldsymbol{H}, 2 m+1)}{S(\boldsymbol{H}, 1)} \mathrm{e}^{-2 \mathrm{i} \xi} F\left(z_{0}, 1\right) .
\end{aligned}
$$

Thus the sequence of the structure amplitudes is here determined by two values: $F\left(z_{0}, 0\right)$ (for the main reflection) and $F\left(z_{0}, 1\right)$ (for the first satellite reflection). Next the physical values of the amplitudes of the modulation function will be found for which the above equations are true.

For the case of one-dimensional modulation function with one harmonic (modulation along the $X$ axis parallel to the vector $\boldsymbol{a}$ ) the amplitude of the modulation function is

$$
\boldsymbol{u}=\boldsymbol{a} u
$$

and we find that

$$
z=H_{1} u
$$

where $H_{1}=2 \pi h+m q$. Thus the modulated structure amplitude for the main reflection and for the first satellite reflection $(m=1)$ is expressed, respectively, as follows:

$$
\begin{aligned}
& F(z, 0)=S(\boldsymbol{H}, 0) J_{0}\left(4 \pi^{2} h u\right) \\
& F(z, 1)=-S(\boldsymbol{H}, 1) J_{1}[2 \pi(2 \pi h+q) u] \exp (-\mathrm{i} \xi) .
\end{aligned}
$$

Above the relation $J_{-m}(z)=(-1)^{m} J_{m}(z)$ has been used.

Differentiating Eq. (13) (in this case $N=1$ ) one obtains the condition for extremum, i.e. the equation on $u$ corresponding to this extremum 


$$
\begin{gathered}
\frac{\partial F(z, m)}{\partial u}=S(\boldsymbol{H}, m)(-1)^{m} \exp (-\mathrm{i} m \xi) 2 \pi(2 \pi h+m q) \\
\times J_{m}^{\prime}[2 \pi(2 \pi h+m q) u]=0 .
\end{gathered}
$$

The solutions of the above equation on $u$ are given by the zeros of the derivative of the Bessel function. These zeros are approximated by the following sequence:

$$
u_{(s)}=\frac{4 s+2 m+1}{8(2 \pi h+m q)},
$$

where $s$ represents an arbitrary integer and covers the infinite number of solutions. One can expect that some of these solutions have physical meaning. The fundamental limitation of these amplitudes of the modulation function is that they should be significantly smaller than the size of the elementary cell. To fulfill this condition one has to fit properly the values of the parameters $q, m, h$, and $s$ in Eq. (39). Let us note that in the above formulae the approximation was made to take the derivatives of the Bessel functions according to the relation (e.g. [11]):

$$
J_{m}^{\prime}(x)=-\sqrt{2 / \pi} \sin [x-(2 m+1) \pi / 4] .
$$

Thus the zeros of $J_{m}^{\prime}(x)$ are equal to $x=\pi s+(2 m+1) \pi / 4$.

Taking into account that $u^{0}$ depends on $m$, one can find that

$$
u^{0}=\frac{4 s+2 m+1}{8(2 \pi h+m q)} \underset{m \rightarrow \infty}{\rightarrow} \frac{1}{4 q}
$$

and

$$
u^{0}=\frac{4 s+2 m+1}{8(2 \pi h+m q)} \underset{m \rightarrow 0}{\rightarrow} \frac{4 s+1}{16 \pi h} .
$$

As it follows from the above relations and taking into account the high order $m$ of satellite reflections, one comes to the conclusion that the amplitude $u$ of the modulation function becomes large for small values of the modulation parameter. It means that this amplitude is not physical. On the other hand, for low $m$ one can always find a physical value of $u$ properly fitting values $s$ and $h$.

\subsection{Physically allowed amplitudes $u_{(s)}$ of the one-harmonic modulation function. Examples}

We show below that there always exist such values of the parameters $q, m, h$, and $s$ for which $u_{(s)}$ have the physical meaning. It is well known from the physical point of view that the amplitude of the modulation function is bounded. Let us assume that this amplitude is lower than $1 \AA$. Taking into account that the lattice parameters in the typical crystals are of the order of $10 \AA$ one can say that in the relative units the maximal amplitude $u_{(s)}$ for fixed $s$ and $m$ has to obey the following normalization condition: 


$$
u_{(s)} \equiv \frac{x_{s}}{2 \pi(2 \pi h+m q)} \leq 0.1,
$$

where $x_{s}$ are zeros of the derivatives of the Bessel functions

$$
J_{m}^{\prime}\left(x_{s}\right)=0 .
$$

Equation (43) gives the exact values of $u_{(s)}$. The first five zeros $x_{s}(s=$ $1, \ldots, 5)$ of $J_{m}^{\prime}(x)$ for $m=1, \ldots, 6$ are given below (Table I).

\section{TABLE I}

The zeros of the derivatives of the Bessel functions $J_{m}^{\prime}(x)$.

\begin{tabular}{c|c|c|r|c|c}
\hline \hline$m$ & $x_{1}$ & \multicolumn{1}{c|}{$x_{2}$} & \multicolumn{1}{c|}{$x_{3}$} & \multicolumn{1}{c|}{$x_{4}$} & $x_{5}$ \\
\hline 1 & 1.841 & 5.331 & 8.536 & 11.706 & 14.864 \\
2 & 3.054 & 6.706 & 9.969 & 13.170 & 16.348 \\
3 & 4.201 & 8.015 & 11.346 & 14.586 & 17.789 \\
4 & 5.317 & 9.285 & 12.682 & 15.964 & 19.196 \\
5 & 6.415 & 10.520 & 13.987 & 17.313 & 20.576 \\
6 & 7.501 & 11.735 & 15.286 & 18.637 & 21.931
\end{tabular}

To illustrate what was said above let us assume first that the modulation parameter $q=0.1$ and $h=3$. In this case the amplitudes of the modulation function for the first five zeros of the derivative of the Bessel function and for the satellite reflections of the order from 1 to 6 are following (Table II).

TABLE II

The amplitudes of the modulation functions for $q=0.1$ and $h=3$.

\begin{tabular}{c|c|c|c|c|c}
\hline \hline$m$ & $\frac{x_{1}}{2 \pi(2 \pi h+m q)}$ & $\frac{x_{2}}{2 \pi(2 \pi h+m q)}$ & $\frac{x_{3}}{2 \pi(2 \pi h+m q)}$ & $\frac{x_{4}}{2 \pi(2 \pi h+m q)}$ & $\frac{x_{5}}{2 \pi(2 \pi h+m q)}$ \\
\hline 1 & 0.015 & 0.045 & 0.072 & 0.098 & 0.125 \\
2 & 0.075 & 0.056 & 0.083 & 0.110 & 0.137 \\
3 & 0.035 & 0.067 & 0.094 & 0.121 & 0.148 \\
4 & 0.044 & 0.077 & 0.105 & 0.132 & 0.159 \\
5 & 0.053 & 0.087 & 0.115 & 0.142 & 0.169 \\
6 & 0.061 & 0.096 & 0.125 & 0.153 & 0.179
\end{tabular}

Thus one can see that there exist a few values of the amplitude of the modulation function fulfilling the condition expressed by Eq. (42).

Let us assume now another five combinations of values of $q$ and $h: q=0.1$ and $h=4$ (Table III), $q=0.1$ and $h=5$ (Table IV), $q=0.5$ and $h=3$ (Table V), $q=0.5$ and $h=4$ (Table VI), $q=0.5$ and $h=5$ (Table VII). 
TABLE III

The amplitudes of the modulation functions for $q=0.1$ and $h=4$.

\begin{tabular}{c|c|c|c|c|c}
\hline \hline$m$ & $\frac{x_{1}}{2 \pi(2 \pi h+m q)}$ & $\frac{x_{2}}{2 \pi(2 \pi h+m q)}$ & $\frac{x_{3}}{2 \pi(2 \pi h+m q)}$ & $\frac{x_{4}}{2 \pi(2 \pi h+m q)}$ & $\frac{x_{5}}{2 \pi(2 \pi h+m q)}$ \\
\hline 1 & 0.012 & 0.034 & 0.054 & 0.074 & 0.094 \\
2 & 0.019 & 0.042 & 0.063 & 0.083 & 0.103 \\
3 & 0.026 & 0.050 & 0.071 & 0.091 & 0.111 \\
4 & 0.033 & 0.058 & 0.079 & 0.100 & 0.120 \\
5 & 0.040 & 0.065 & 0.087 & 0.107 & 0.128 \\
6 & 0.046 & 0.073 & 0.095 & 0.115 & 0.136
\end{tabular}

In this case exist more values of the amplitude of the modulation function, lower than 0.1 , than in previous example.

TABLE IV

The amplitudes of the modulation functions for $q=0.1$ and $h=5$

\begin{tabular}{c|c|c|c|c|c}
\hline \hline$m$ & $\frac{x_{1}}{2 \pi(2 \pi h+m q)}$ & $\frac{x_{2}}{2 \pi(2 \pi h+m q)}$ & $\frac{x_{3}}{2 \pi(2 \pi h+m q)}$ & $\frac{x_{4}}{2 \pi(2 \pi h+m q)}$ & $\frac{x_{5}}{2 \pi(2 \pi h+m q)}$ \\
\hline 1 & 0.009 & 0.027 & 0.043 & 0.059 & 0.075 \\
2 & 0.015 & 0.034 & 0.050 & 0.066 & 0.082 \\
3 & 0.021 & 0.040 & 0.057 & 0.073 & 0.089 \\
4 & 0.027 & 0.046 & 0.063 & 0.080 & 0.096 \\
5 & 0.032 & 0.052 & 0.070 & 0.086 & 0.103 \\
6 & 0.037 & 0.058 & 0.076 & 0.093 & 0.109
\end{tabular}

In this case only two values of the amplitude have no physical meaning, the first one for $m=5$ and $s=5$, the second one for $m=6$ and $s=5$.

TABLE V

The amplitudes of the modulation functions for $q=0.5$ and $h=3$.

\begin{tabular}{c|c|c|c|c|c}
\hline \hline$m$ & $\frac{x_{1}}{2 \pi(2 \pi h+m q)}$ & $\frac{x_{2}}{2 \pi(2 \pi h+m q)}$ & $\frac{x_{3}}{2 \pi(2 \pi h+m q)}$ & $\frac{x_{4}}{2 \pi(2 \pi h+m q)}$ & $\frac{x_{5}}{2 \pi(2 \pi h+m q)}$ \\
\hline 1 & 0.015 & 0.044 & 0.070 & 0.096 & 0.122 \\
2 & 0.024 & 0.054 & 0.080 & 0.106 & 0.131 \\
3 & 0.033 & 0.063 & 0.089 & 0.114 & 0.139 \\
4 & 0.041 & 0.071 & 0.097 & 0.122 & 0.147 \\
5 & 0.048 & 0.078 & 0.104 & 0.129 & 0.153 \\
6 & 0.055 & 0.085 & 0.111 & 0.136 & 0.160
\end{tabular}

In this case there exists one more combination of $m$ and $s$ giving the physical meaning, than in the case with $q=0.1$ and $h=3$. 
TABLE VI

The amplitudes of the modulation functions for $q=0.5$ and $h=4$.

\begin{tabular}{c|c|c|c|c|c}
\hline \hline$m$ & $\frac{x_{1}}{2 \pi(2 \pi h+m q)}$ & $\frac{x_{2}}{2 \pi(2 \pi h+m q)}$ & $\frac{x_{3}}{2 \pi(2 \pi h+m q)}$ & $\frac{x_{4}}{2 \pi(2 \pi h+m q)}$ & $\frac{x_{5}}{2 \pi(2 \pi h+m q)}$ \\
\hline 1 & 0.011 & 0.033 & 0.053 & 0.073 & 0.092 \\
2 & 0.019 & 0.041 & 0.061 & 0.080 & 0.100 \\
3 & 0.025 & 0.048 & 0.068 & 0.087 & 0.106 \\
4 & 0.031 & 0.054 & 0.074 & 0.094 & 0.113 \\
5 & 0.037 & 0.061 & 0.081 & 0.100 & 0.119 \\
6 & 0.042 & 0.066 & 0.086 & 0.105 & 0.124
\end{tabular}

In this case a lot of the values of the amplitude have physical meaning.

TABLE VII

The amplitudes of the modulation functions for $q=0.5$ and $h=5$.

\begin{tabular}{c|c|c|c|c|c}
\hline \hline$m$ & $\frac{x_{1}}{2 \pi(2 \pi h+m q)}$ & $\frac{x_{2}}{2 \pi(2 \pi h+m q)}$ & $\frac{x_{3}}{2 \pi(2 \pi h+m q)}$ & $\frac{x_{4}}{2 \pi(2 \pi h+m q)}$ & $\frac{x_{5}}{2 \pi(2 \pi h+m q)}$ \\
\hline 1 & 0.009 & 0.027 & 0.043 & 0.058 & 0.074 \\
2 & 0.015 & 0.033 & 0.049 & 0.065 & 0.080 \\
3 & 0.020 & 0.039 & 0.055 & 0.071 & 0.086 \\
4 & 0.025 & 0.044 & 0.060 & 0.076 & 0.091 \\
5 & 0.030 & 0.049 & 0.066 & 0.081 & 0.097 \\
6 & 0.035 & 0.054 & 0.071 & 0.086 & 0.101
\end{tabular}

In this case only one value of the amplitude has no physical meaning (for $m=6$ and $s=5)$.

Equation (42) can be rewritten in the following form:

$$
\frac{10 x_{s}}{2 \pi m} \leq q+\frac{2 \pi h}{m}
$$

which gives the general relationship between the allowed values of $q$ and $h$ for the five zeros mentioned above and for the different $m$ values. The corresponding values are presented in Table VIII.

TABLE VIII

Relationship between the allowed values of $q$ and $h$.

\begin{tabular}{c|r|c|c|c|c|c}
\hline \hline$m$ & & $\frac{10 x_{1}}{2 \pi m}$ & $\frac{10 x_{2}}{2 \pi m}$ & $\frac{10 x_{3}}{2 \pi m}$ & $\frac{10 x_{4}}{2 \pi m}$ & $\frac{10 x_{5}}{2 \pi m}$ \\
\hline 1 & $q+2 \pi h>$ & 2.93 & 8.49 & 13.59 & 18.64 & 23.67 \\
2 & $q+\pi h>$ & 2.43 & 5.34 & 7.94 & 10.49 & 13.02 \\
3 & $q+2 \pi h / 3>$ & 2.23 & 4.25 & 6.02 & 7.74 & 9.44 \\
4 & $q+\pi h / 2>$ & 2.12 & 3.70 & 5.05 & 6.36 & 7.64 \\
5 & $q+2 \pi h / 5>$ & 2.04 & 3.35 & 4.45 & 5.51 & 6.55 \\
6 & $q+\pi h / 3>$ & 1.99 & 3.11 & 4.05 & 4.95 & 5.82
\end{tabular}


In Table VIII the conditions are presented which concern the physical values of the modulation parameter $q$ for the arbitrary $h$ (fulfilling the above inequalities). Let us note that when - for instance - the limitation in Eq. (42) was twice lower (it means 0.05 instead of 0.1 ), the physically reasonable value of the index $h$ should be greater than 6 . One has to point out that the analogous way of thinking applies also for three dimensions.

\section{Discussion}

As it was shown in Sect. 2, the structure amplitude of the displacively modulated structure tends to zero both for small and big values of the amplitudes of the modulation function. Therefore a conclusion was made that there should exist certain values of the amplitudes of the modulation function for which the modulus of the structure amplitude assumes a maximum value. For such amplitudes of the modulation function the intensity of an arbitrary satellite reflection turns out to be a function of the intensities of both the closest main reflection and the intensities of the finite set of the neighbor satellite reflections. In Sect. 3 the above considerations are applied to the case of one-dimensional modulation with the modulation function consisting of one harmonic. The explicit form was found of the amplitudes $u_{(s)}$ of the modulation function for which the modulus of the structure amplitude assumes a maximum value. $u_{(s)}$ make a series indexed by the arbitrary integer $s$. However, not all the amplitudes $u_{(s)}$ have a physical meaning. The only physical values of $u_{(s)}$ have to fulfill - at least - the following relation:

$$
u_{(s)}<0.1 \text {. }
$$

Our considerations concern the modulated structures with the continuous modulation functions only. As the quasicrystals are concerned, they possess the symmetry elements excluding the translational symmetry, although they reveal a long-range ordering. Also their diffraction reflections can be described with the aid of more than three integer indices. Therefore other methods are needed for the quasicrystal structure analysis (e.g. [12]).

\section{Appendix}

\section{A.1. Method of a stationary phase}

Method of a stationary phase describes the asymptotic behavior of the oscillatory integral

$$
\int_{M} f(x) \mathrm{e}^{\mathrm{i} z \Phi(x)} \mathrm{d} x
$$

for very large $z$. The above integral has the following value (if $z \rightarrow \infty)$ ([13], p. 6): 


$$
\begin{aligned}
\int_{M} f(x) \mathrm{e}^{\mathrm{i} z \Phi(x)} \mathrm{d} x=\left(\frac{2 \pi}{z}\right)^{n / 2} \sum_{x_{\mathrm{c}}} \mathrm{e}^{\pi \mathrm{i} \operatorname{sgn} H\left(x_{\mathrm{c}}\right)} \frac{\mathrm{e}^{\mathrm{i} z \Phi\left(x_{\mathrm{c}}\right)} f\left(x_{\mathrm{c}}\right)}{\sqrt{\left|\operatorname{det} H\left(x_{\mathrm{c}}\right)\right|}} \\
+\mathrm{O}\left(z^{-n / 2-1}\right),
\end{aligned}
$$

where $x_{\mathrm{c}}$ are critical points of $\Phi(x): \Phi^{\prime}\left(x_{\mathrm{c}}\right)=0, H\left(x_{\mathrm{c}}\right)$ is the Hessian of $\Phi$ at $x_{\mathrm{c}}$ :

$$
H\left(x_{\mathrm{c}}\right)=\left[\frac{\partial^{2} \Phi}{\partial x_{i} \partial x_{j}}\left(x_{\mathrm{c}}\right)\right],
$$

and $n$ is the dimension of the manifold $M$. In the case under consideration $n$ is equal to unity and the manifold $M$ is a circle.

\section{A.2. Amplitudes}

Equation (19) gives the recurrence relation between different structure amplitudes. Let us rewrite this equation (omitting the symbol $\boldsymbol{z}_{0}$ ) as follows:

$$
F(m+H)=C(m, H) F(m-H),
$$

where $H=1, \ldots, N$ and

$$
C(m, a)=\frac{S(m+a)}{S(m-a)} \exp \left(-2 \mathrm{i} \xi_{a}\right) .
$$

Thus we have $N$ equations for amplitudes $F$. These equations are related between each other. They have the form

$$
\begin{gathered}
F(m+1)=C(m, 1) F(m-1), \\
F(m+2)=C(m, 2) F(m-2), \\
\vdots \\
F(m+N)=C(m, N) F(m-N) .
\end{gathered}
$$

From the first equation (A2.3.1) one obtains the following relations:

$$
\begin{aligned}
& F(2 m)=\prod_{l=1}^{m} C(2 l-1,1) F(0), \\
& F(2 m+1)=\prod_{l=1}^{m} C(2 l, 1) F(1)
\end{aligned}
$$

for $m \geq 1$.

From the second equation (A2.3.2) one obtains the relations as follows:

$$
F(4 m)=\prod_{l=1}^{m} C(4 l-2,2) F(0),
$$




$$
\begin{aligned}
& F(4 m+1)=\prod_{l=1}^{m} C(4 l-1,2) F(1), \\
& F(4 m+2)=\prod_{l=1}^{m} C(4 l, 2) F(2), \\
& F(4 m+3)=\prod_{l=1}^{m} C(4 l+1,2) F(3)
\end{aligned}
$$

for $m \geq 2$. And so on.

Finally from the last equation $(\mathrm{A} 2.3 . \mathrm{N})$ one obtains the following relations:

$$
\begin{gathered}
F(2 N m)=\prod_{l=1}^{m} C(2 N l-N, N) F(0), \\
F(2 N m+1)=\prod_{l=1}^{m} C[2 N l-(N-1), N] F(1), \\
\vdots \\
F(2 N m+2 N-1)=\prod_{l=1}^{m} C[2 N l+(N-1), N] F(2 N-1)
\end{gathered}
$$

for $m \geq N$.

From the above solutions one can see that there exist $2 N$ independent quantities $F$ :

$$
\{F(0), F(1), \ldots, F(2 N-1)\},
$$

by which any other structure amplitude can be expressed.

\section{A.3. Expansion for small $u$}

If the amplitude of modulation function approaches zero (in the one-harmonic case), then the structure amplitude assumes the form (e.g. [3]):

$$
F(\boldsymbol{z}, m) \underset{u_{\alpha 1} \rightarrow 0}{\sim} S(\boldsymbol{H}, m)(-1)^{m} \exp (-\mathrm{i} m \xi) \frac{\left(\pi H u_{\alpha 1}\right)^{m}}{\Gamma(1+m)},
$$

because the expansion of the Bessel function for $m$ fixed and $u \rightarrow 0$ is given by [11]:

$$
J_{m}\left(u_{\alpha 1}\right) \underset{u_{\alpha 1} \rightarrow 0}{\sim} \frac{\left(\frac{u_{\alpha 1}}{2}\right)^{m}}{\Gamma(1+m)} .
$$

If $u$ approaches zero, then the structure amplitude decreases proportionally to $u_{\alpha 1}^{m}$. Let us note that the structure amplitude becomes weaker when the order $m$ of the satellite becomes higher whereas for $m$ equal to zero the structure amplitude describes the main reflections. For the case with $N$ harmonics, when $u_{\alpha 1}, \ldots, u_{\alpha N}$ 
tend to zero, the factor $g$ in the amplitude $F$ assumes the form

$$
g\left(u_{\alpha 1}, \ldots, u_{\alpha N}\right)_{u_{\alpha 1}, \ldots, u_{\alpha N} \rightarrow 0}^{\rightarrow} \frac{1}{2 \pi} \int_{0}^{2 \pi} \exp (\mathrm{i} m x) \mathrm{d} x=\delta_{m, 0} .
$$

\section{References}

[1] P.M. de Wolff, T. Janssen, A. Janner, Acta Crystallogr. A 37, 625 (1981).

[2] J.M. Perez-Mato, G. Madariaga, Solid State Commun. 58, 105 (1986).

[3] W.A. Paciorek, D. Kucharczyk, Acta Crystallogr. A 41, 462 (1985).

[4] I. Aramburu, G. Madariaga, J.M. Perez-Mato, Acta Crystallogr. A 53, 334 (1997).

[5] I. Aramburu, G. Madariaga, J.M. Perez-Mato, Acta Crystallogr. A 53, 329 (1997).

[6] H. Böhm, Acta Crystallogr. A 31, 622 (1975).

[7] W. van Aalst, J. den Hollander, W.J.A.M. Peterse, P.M. de Wolff, Acta Crystallogr. B 32, 47 (1976).

[8] T. Janssen, A. Janner, A. Looijenga-Vos, P.M. de Wolff, International Tables of Crystallography, Vol. c, Kluwer Academic Publ., Dordrecht 1992, p. 797.

[9] S. van Smaalen, Ph.D. thesis, Rijksuniversiteit, Groningen 1985.

[10] W.A. Paciorek, G. Chapuis, Acta Crystallogr. A 50, 194 (1993).

[11] M. Abramowitz, I.A. Stegun, Handbook of Mathematical Functions with Formulas, Graphs and Mathematical Tables, National Bureau of Standards Applied Mathematics Series, Washington D.C. 1964.

[12] G. Urban, J. Wolny, Philos. Mag. 84, 2905 (2004).

[13] V. Guillemin, S. Sternberg, Geometrics Asymptotics, American Mathematical Society, Providence 1977. 